\title{
Anti-Ri-related Possible Autoimmune Encephalitis may Cause Intellectual and Developmental Disabilities in Pediatric Patients: A Case Report
}

\section{Zhijie Zhang}

Shanghai Jiaotong University School of Medicine Xinhua Hospital Jing Wu

Shanghai Jiaotong University School of Medicine Xinhua Hospital

Danfen Xu

Shanghai Jiaotong University School of Medicine Xinhua Hospital

Shennan Zhao

Shanghai Jiaotong University School of Medicine Xinhua Hospital

Di Lian

Shanghai Jiaotong University School of Medicine Xinhua Hospital

Dandan Zhang

Shanghai Jiaotong University School of Medicine Xinhua Hospital

Ling Li ( $\sim$ liling@xinhuamed.com.cn )

Shanghai Jiaotong University School of Medicine Xinhua Hospital https://orcid.org/0000-0002-09773822

\section{Case report}

Keywords: intellectual disabilities, Ri antibody, autoimmune encephalitis

Posted Date: June 1st, 2021

DOl: https://doi.org/10.21203/rs.3.rs-547839/v1

License: (a) (1) This work is licensed under a Creative Commons Attribution 4.0 International License. Read Full License 


\section{Abstract}

Objective: To describe two rare pediatric cases in which anti-Ri autoantibody-positive (Type II antineuronal nuclear antibody; ANNA-2-positive) autoimmune encephalitis occurred with intellectual and development disabilities..

Case presentation: We presented the clinical manifestations, etiological evaluation, and intellectual assessment of two ANNA-2-positive children and the clinical improvement after empirical immunotherapy. A 2-year-old girl with hyperactivity, agitation, and hand tremors and a 6-month-old boy who had dystonia were both diagnosed with intellectual and development disability. Both children tested positive for ANNA-2 and the serological inflammatory markers. Considering possible Ri antibody associated autoimmune encephalitis, empirical immunotherapy was done for both patients, intellectual disability of them improved to some extent.

Conclusions: Although ANNA-2 has been mostly reported in adults with paraneoplastic syndrome, we report two ANNA-2-positive pediatric cases diagnosed as possible autoimmune encephalitis due to viral infections. These cases suggested that disruption of the immune system due to viral infection may lead to ANNA-2 positive status and contribute to intellectual and development disability in pediatric cases.

\section{Introduction}

Intellecutual and development disability patients are at an increased risk for a variety of comorbidities, including epilepsy or convulsive disorders and bring large economic burden. Autoimmune encephalitis is proven to cause intellectual dissability in pediatric. Among the antibody cause autoimmune encephalitis, $\mathrm{Ri}$ is a neuron-specific RNA-binding protein widely expressed in the CNS (1). An association has been reported between anti-Ri autoantibody (Type II anti-neuronal nuclear antibody, ANNA-2) and opsoclonusmyoclonus syndrome, ataxia, dementia, short-term memory loss, and paraneoplastic cerebellar degeneration in adults with cancers $(2,3)$. However, the clinical manifestations and epidemiology in ANNA-2-positive pediatric patients have not been reported. Herein, we report two pediatric cases with possible association between ANNA-2 and intellecutal and development disability. .

\section{Case Reports}

\section{Case 1}

A two-year-old girl presented with a history of delayed motor skills and language development. She could lift her head and hold its position at 6 months, sit in a tripod fashion without support at 12 months, stand with support at 13 months, and walk with assistance at 18 months. There had been no improvement in language after she could say "mum" and "dad" at 18 months. Additionally, she had symptoms of hyperactivity and agitation. Family, prenatal, perinatal, and neonatal histories were normal. Pathologic reflexes were negative. 
After admission, laboratory tests showed lymphocytosis $\left(4.81 \times 10^{9}\right.$ cells/L, normal: $\left.1.6-3.2 \times 10^{9}\right)$ and herpes simplex virus (HSV) infection (serologically reactive HSV-1/2 IgM 1.25; normal: < 1.00). Inflammation was also present, based on elevated tumor necrosis factor alpha (TNF-a; $16.10 \mathrm{pg} / \mathrm{mL}$, normal: <2.8), interleukin-2 (IL-2) receptor $(794.0 \mathrm{U} / \mathrm{mL}$, normal: $100-500)$, and CD4+ (1972.74 cells/ $\mu \mathrm{L}$, normal: $500-1200)$ and CD19 + cells (1336.82 cells/ $\mu \mathrm{L}$, normal: 103-581). Thyroid function and its associated antibody tests were normal. Results of gas chromatography-tandem mass spectrometry of blood, urine, and blood lactic acid were normal, but anti-dsDNA was found in the serum.

MRI showed bilateral T2 hyperintensity within the occipital lobes (Fig. 1-A). EEG results did not show any abnormalities. Lumbar puncture results were unremarkable, with an absence of oligoclonal bands and normal levels of IgG, IgA, and IgM. HSV and cytomegalovirus (CMV) were not seen in real-time PCR of the CSF. While serum immunofluorescence detected ANNA-2, it was not found in the CSF. Gesell developmental diagnosis scale on day 8 of hospitalization, showed delays in adaptability, gross motor skills, fine motor skills, language skills, and social ability. Auditory and visual evoked potentials showed prolongation of the P100 latency and increased hearing thresholds bilaterally.

The patient received acyclovir ( $30 \mathrm{mg} / \mathrm{kg} /$ day, 14 days) for HSV infection. On day 6 , sporadic irregular tremors were observed in both hands, which worsened when holding things and being emotional. Immunosuppressive therapy for possible autoimmune encephalitis was administered as follows: intravenous immunoglobulin ( $1 \mathrm{~g} / \mathrm{kg} /$ day for 2 days) and methylprednisolone $(20 \mathrm{mg} / \mathrm{kg} /$ day for 5 days, followed by $10 \mathrm{mg} / \mathrm{kg} /$ day for 5 days, $5 \mathrm{mg} / \mathrm{kg} /$ day for 5 days, and $2 \mathrm{mg} / \mathrm{kg} /$ day for 5 days). During treatment, CMV DNA was detected in her urine. Hence, the acyclovir treatment was followed by 14 days of ganciclovir. After treatment, HSV IgM decreased to normal levels (0.70). However, the hyperintensity seen on the previous MRI showed no improvement after treatment (Fig. 1-B). While there was little improvement in her language and motor skills, the hyperactivity and agitation symptoms decreased dramatically and the tremors reduced in severity.

\section{Case 2}

A six-month-old male infant presented with major motor development retardation. He could lift his head but not hold the position. Family and birth history were normal. A neurological examination revealed symmetrical increased muscular tension in the lower extremities. Pathologic reflexes were negative on both sides. Sucking reflex, cuddling reflex, parachute reflex, and foraging reflex were negative. After being admitted to our hospital, we observed more than 10 episodes of lateral body dystonia with crying daily.

Upon admission, laboratory results showed lymphocytosis $\left(10 \times 10^{9}\right.$ cells $\left./ \mathrm{L}\right)$ and $\mathrm{CMV}$ infection (serological CMV IgM 27.4 U/mL). CMV DNA was found in the urine but not in serum. Elevated TNF-a $(14.6 \mathrm{pg} / \mathrm{mL}), \mathrm{IL}-2$ receptor $(1359 \mathrm{U} / \mathrm{mL})$, and CD4+ (4300.51/ $\mathrm{LL})$ and CD19 + cells $(2262.08 / \mu \mathrm{L})$ demonstrated inflammation. Like case 1, test results for thyroid function and its associated antibodies, blood, urine, and blood lactic acid were normal. Serum immunofluorescence was positive for ANNA-2. 
Tests for HSV and CMV antibodies, other autoimmune antibodies, oligoclonal bands, and immunoglobulins were negative in the CSF. Long-term EEG did not show any abnormalities. MRI and CT were unremarkable. Intelligence assessment was performed with Bayley scales, indicating motor and cognitive developmental delays. The patient failed the distortion product otoacoustic emission test for newborn hearing screening.

He had received ganciclovir (10 mg/kg/day, 14 days) for the CMV infection before admission to our hospital. We continued ganciclovir for another 14 days and administered immunosuppressive therapy for possible autoimmune encephalitis including intravenous immunoglobulin and methylprednisolone. Additionally, oxcarbazepine and benzhexol were administered to treat dystonia. During treatment, there was minor improvement in gross motor function, but the dystonia showed no alleviation. To exclude the possibility of a concurrent tumor, serological tumor markers were evaluated and PET-CT was performed (Fig. 3), which showed reduced fluorodeoxyglucose uptake bilaterally in the parietal lobes. We performed exome sequencing and copy number variation analysis, which detected no significant genetic alteration. Serological CMV IgM persisted throughout the treatment. After discharge, the patient was advised to go to a rehabilitation center for regular cognitive and physical therapy. His muscular tension was alleviated with therapy, but there was little improvement in gross motor skills and dystonia.

\section{Discussion}

We presented two very rare cases of pediatric patients being diagnosed with anti-Ri autoantibody-related possible autoimmune encephalitis. Both ANNA-2-positive patients had mental retardation with evidence of primary CMV and HSV infection on serology. Case 1 showed psychiatric symptoms, tremors, and cognitive impairment, while case 2 showed motor development delay and dystonia. Both pediatric cases met the symptomology of possible autoimmune encephalitis, which was supported by their laboratory results (4). ANNA-2 is the most common intracellular neuronal antibody marker(5). Furthermore, classic opsoclonus-myoclonus syndrome, psychosis, dyskinesia, and weakness can be the presenting features of ANNA-2-related autoimmune encephalitis. The symptoms the two pediatric cases in this report showed tremors, psychosis and dytonia. These findings provide evidence that support these patients having ANNA-2-associated possible autoimmune encephalitis. Cognitive impairment and development delay are characteristic features in pediatric autoimmune encephalitis, as seen in our cases(4). It is possible that the ANNA-2-associated possible autoimmune encephalitis contributed to the Cognitive impairment and development delay in the two cases.

The first question of interest is how and why ANNA-2, a well-known paraneoplastic antibody, was present without cancer in pediatric patients. It is well known that CMV and HSV can lie latent in the mucosa and neurons $(5,6)$. Consequently, recent studies have indicated that an imbalance between immunomicrobiology and the immune system could impair the quality of life in the elderly (7), and immune dysregulation in post-HSV encephalitis could cause autoimmune encephalitis (8). Moreover, pediatric autoimmune neuropsychiatric disorders associated with Streptococcal infections can be 
postinfectious and have symptoms similar to our patients (9). Hence, we postulated that pediatric patients could also have immune dysregulation by viral infections.

The second question is why immunosuppressive therapy did not work as expected for ANNA-2associated autoimmune encephalitis. We propose two possible reasons. First, $\mathrm{Ri}$ is an intracellular protein, which has been proven insensitive to humoral immunotherapy (10). Second, ANNA-2 may not be the major causal factor for the neurological deficits in the two cases; cytotoxic T cells, manic B cells, or NK cells may be the real cause of the neurological deficits. However, we believe that ANNA-2 could be evidence of immune dysregulation in patients with CMV and HSV infections, so it is important to consider this possibility in pediatric patients with viral infections.

\section{Conclusion}

Overall, this case report suggests a possible association between ANNA-2 and neurodevelopment disorders in pediatric patients; however, more cases are needed to support a causal relationship. These two cases suggest the need to reevaluate potential neurological symptoms in latent and subclinical infections of CMV and HSV. Furthermore, this report reminds clinicians to consider immune dysregulation in pediatric patients with intellectual and developmental disabilities.

\section{Abbreviations}

CMV: cytomegalovirus

CT: computerized tomography

HSV: herpes simplex virus

MRI: magnetic resonance

PET-CT: Positron Emission Tomography-Computed Tomography

TNF-a: tumor necrosis factor-a

\section{Declarations}

\section{Ethical Approval and Consent to participate}

Patients in the case reports signed the written informed consent(Supplementary Material)

\section{Consent for publication}

Two cases consent to publication was provided by all participants. 


\section{Availability of data and materials}

All data generated or analysed during this study are included in this published article.

\section{Competing interests}

The authors declare no competing interests.

\section{Funding}

This work was funded by the National Natural Science Foundation of China $(82071350,81873760)$.

\section{Authors' contributions}

ZJZ and JW interpreted the patient data and completed the manuscript,They both were major contributor in writing the manuscript. SNZ, DFX, DDZ, DL helped collection the patient data. LL guided the project and reviewed the paper. All authors read and approved the final manuscript

\section{Acknowledgements}

The authors sincerely thank the families that contributed to this study.

\section{References}

1. Simard C, Vogrig A, Joubert B, Joubert, S. Muniz-Castrillo, G. Picard, V. Rogemond, F. , et al. Clinical spectrum and diagnostic pitfalls of neurologic syndromes with Ri antibodies. Neurol Neuroimmunol Neuroinflamm 2020; 7(3):e699.

2. Takkar A, Mehta S, Gupta N, Bansal S and V. Lal . Anti- RI antibody associated progressive supranuclear palsy like presentation in a patient with breast carcinoma. $\mathrm{J}$ Neuroimmunol 2020;347:577345.

3. Tesseki K, Kataoka H, Terashima M, Kawahara, T. Taoka, K. Tanaka et al. Biphasic paraneoplastic brainstem encephalitis associated with anti-Ri antibody. J Neurooncol 2010;100(1):141-143.

4. Cellucci, T., H. Van Mater, F. Graus, E. Muscal, W. Gallentine, M. S. Klein-Gitelman, et al. Clinical approach to the diagnosis of autoimmune encephalitis in the pediatric patient. Neurol Neuroimmunol Neuroinflamm 2020;7(4):e730.

5. 5. Nikolich-Zugich, J., L. Cicin-Sain, D. Collins-McMillen, S. Jackson, A. Oxenius, J. Sinclair, C囚et al. Advances in cytomegalovirus (CMV) biology and its relationship to health, diseases, and aging. 
Geroscience 2020;42(2):495-504.

6. 6. Whitley R, Baines J. Clinical management of herpes simplex virus infections: past, present, and future. F1000Res 2018;7:F1000 Faculty Rev-1726.

7. 7. Franceschi C, Campisi J. Chronic inflammation (inflammaging) and its potential contribution to age-associated diseases. J Gerontol A Biol Sci Med Sci 2014;69 Suppl 1:S4-9.

8. H, Alexopoulos, Akrivou S, Mastroyanni S, Antonopoulou M, Dinopoulos A, Giorgi M, et al. Postherpes simplex encephalitis: a case series of viral-triggered autoimmunity, synaptic autoantibodies and response to therapy. Ther Adv Neurol Disord 2018;11:1756286418768778.

9. Pollak TA, Lennox BR, Müller S, Me Benros, H Pruss, Vanelstl Tebartz, et al. Autoimmune psychosis: an international consensus on an approach to the diagnosis and management of psychosis of suspected autoimmune origin. Lancet Psychiatry 2020;7(1):93-108.

10. Varley J, Taylor J, Irani SR. Autoantibody-mediated diseases of the CNS: Structure, dysfunction and therapy. Neuropharmacology 2018;132:71-82.

\section{Figures}

T1

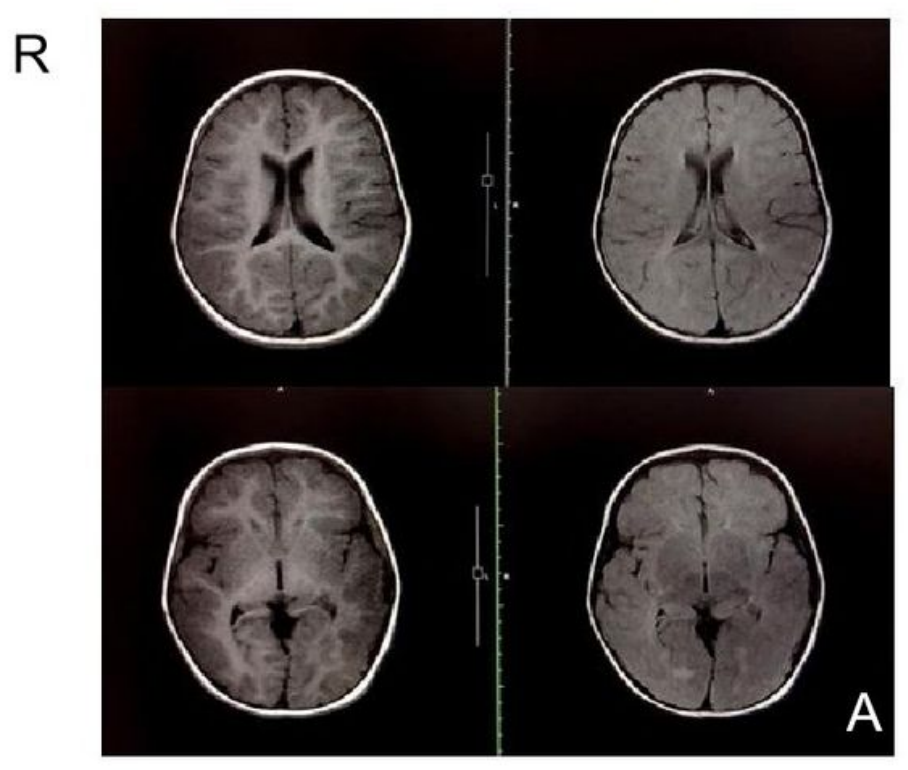

T1

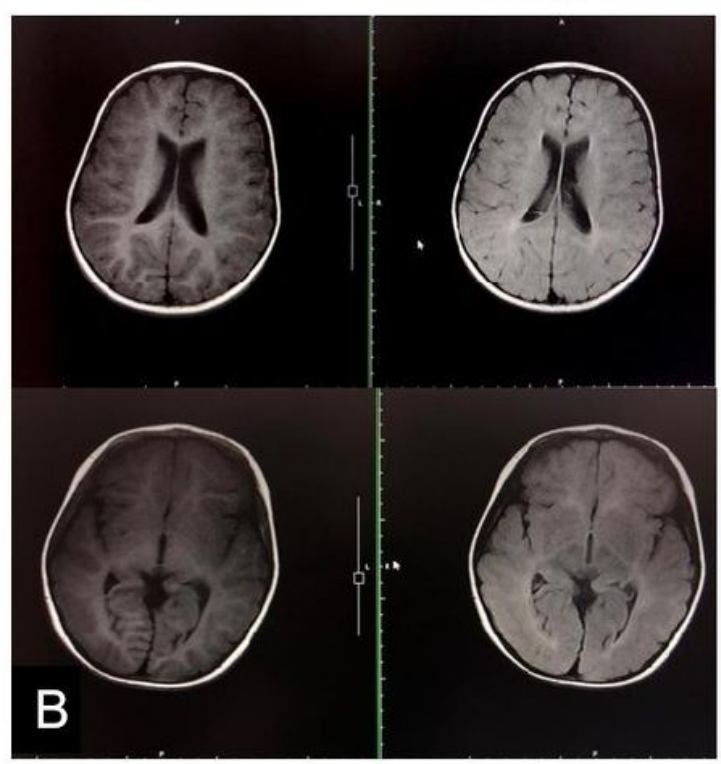

Figure 1

A:MRI of case 1 with bilateral T2 hyperintensity within the occipital lobes $₫ \mathrm{~B}: M R I$ of case 1 after treatment 


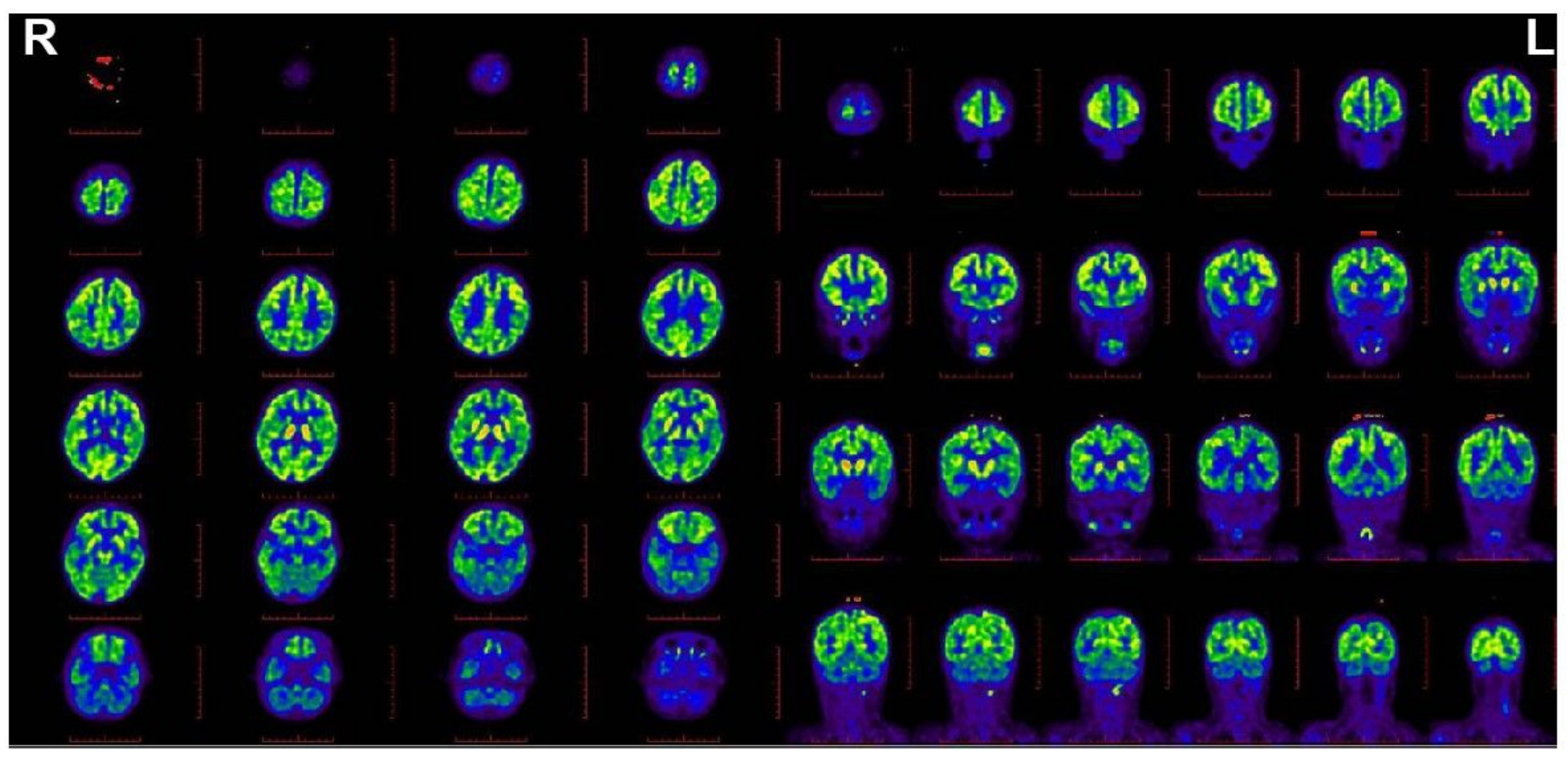

Figure 2

PET-CT of case 2 showing reduced fluorodeoxyglucose uptake in the bilateral parietal cortex.

\section{Supplementary Files}

This is a list of supplementary files associated with this preprint. Click to download.

- PATIENT1CONSTENTFORM.pdf

- PATIENT2CONSTENTFORM.pdf 